
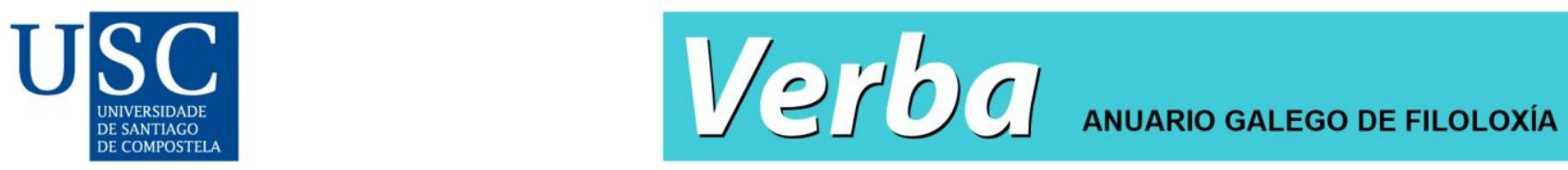

Verba: Anuario Galego de Filoloxía, 48, 2021. ISSN: 2174-4017

https://doi.org/10.15304/verba.48.7088

Revista de libros

\title{
Jesús Pena (ed.) (2017): Procesos morfológicos. Zonas de interferencia, Anexo 76 de Verba, Santiago de Compostela: Universidade de Santiago de Compostela, 239 pp.
}

\author{
Elena Felíu Arquiola ${ }^{1}$ \\ 1Universidad de Jaén, España
}

En mayo de 2016 tuvo lugar en la Universidade de Santiago de Compostela el XII Encuentro de Morfólogos, reunión científica que se viene celebrando anualmente desde 2005 en diversas universidades españolas. Si en ediciones anteriores se había abordado, entre otros temas, el estudio de los afijos como unidades léxicas (VIII Encuentro de Morfólogos: los afijos: variación, rivalidad y representación, Universitat Pompeu Fabra, 2012) o los límites de la morfología con otros componentes gramaticales y otras disciplinas (XI Encuentro de Morfólogos: Los lindes de la morfología, Universitat Autònoma de Barcelona, 2015), en esta duodécima edición el tema elegido por el comité organizador - coordinado por Jesús Pena y María José Rodríguez Espiñeira e integrado por miembros del proyecto MORFOGEN y del grupo de investigación Gramática del español (USC GI-1372) - fueron los procesos morfológicos y las fronteras entre ellos, tal como se refleja en el título del volumen reseñado, que coincide con el del Encuentro.

Se trata, sin duda, de un tema de gran relevancia tanto para la teoría morfológica como para la morfología descriptiva del español y de otras lenguas romances, que supone además una toma de postura teórica, pues se presta atención a los procesos en sí mismos (derivación, composición, acronimia, etc.) y no tanto a los exponentes o a los resultados de dichos procesos. Igualmente, desde nuestro punto de vista constituye un acierto poner énfasis principalmente en las zonas de interferencia o de solapamiento entre los procesos morfológicos, esto es, centrarse no tanto en la caracterización de cada proceso en sí mismo, sino en su relación con los demás, ya que indagar en esos límites, a veces difusos, puede conducirnos a un conocimiento más preciso de cada tipo de proceso.

La monografía Procesos morfológicos. Zonas de interferencia, editada por Jesús Pena, consta de una presentación y diez estudios: por una parte, cinco artículos que versan sobre el tema principal del volumen y que se corresponden con las contribuciones de los cinco ponentes invitados al XII Encuentro, todos ellos renombrados especialistas en morfología del español o de otras lenguas romances; por otra, cinco notas o trabajos más breves que se corresponden con una selección de 
los pósteres presentados en el Encuentro, algunos de los cuales también abordan cuestiones relativas a los procesos morfológicos y sus interferencias.

Como primer artículo encontramos el trabajo de Graça Rio-Torto (Universidade de Coimbra), titulado "Composição, afixação, sintagmação", cuyo objetivo es explorar los límites internos y externos de la composición. Tras una presentación de las principales contribuciones que la gramaticografía románica - principalmente española y portuguesa- ha realizado a la caracterización de la composición, la autora se centra en los límites de la composición neoclásica respecto de la prefijación y de la sufijación. Finalmente, la parte más extensa del estudio está dedicada a delimitar las fronteras entre la composición sintagmática, especialmente el esquema compositivo [NprepN] $]_{\mathrm{N}}$ (arma de fuego), y los sintagmas libres [N1 [PrepN2]sp] (mesa de madera), tanto en portugués como en español. Para ello, la autora aplica una serie de criterios diferenciadores empleados habitualmente en la bibliografía, entre los que se encuentran los siguientes: grado de idiomaticidad, grados de opacidad formal, procesamiento holístico de la expresión pluriverbal, motivación semántico-pragmática y paradigmaticidad.

El trabajo de David Serrano-Dolader (Universidad de Zaragoza), titulado "La parasíntesis como proceso lexicogenético (no tan) peculiar", comienza con un recorrido histórico por el término y el concepto de parasíntesis. Posteriormente se abordan los límites borrosos entre la parasíntesis y otros procedimientos de formación de palabras como la prefijación, la sufijación, la conversión, la circunfijación y la composición. Al tratar cada una de estas zonas de interferencia, el autor discute pormenorizadamente cuestiones tradicionalmente conflictivas y aporta su propuesta para cada caso. Algunos de estos aspectos polémicos son, por ejemplo, la función transcategorizadora o no de los prefijos en los verbos parasintéticos; la consideración del sufijo de infinitivo o de la vocal temática como sufijo derivativo en casos como embarcar o encarcelar; la consideración de prefijo y sufijo en las formaciones parasintéticas como circunfijo o no, etc. Finalmente, el autor presenta una nueva concepción de la denominada "parasíntesis en composición" que va más allá de formaciones poco sistemáticas como corchotaponero o doceañista. A partir de la definición de la parasíntesis como un proceso lexicogenético particularizado por el hecho de que combina solidariamente procedimientos diversos, Serrano-Dolader propone ampliar el concepto de parasíntesis en composición en dos sentidos: por una parte, para incluir formaciones de otros idiomas que no cuentan con esta tradición terminológica (blue-eyed en inglés o rothaarig en alemán); por otra, para incluir formaciones del español como lavacoches o limpiabotas, que se generarían por la actuación de dos procedimientos de forma solidaria: composición y derivación de verbo a nombre (en el caso del primer constituyente del compuesto).

El tercer artículo corre a cargo de Josefa Martín García (Universidad Autónoma de Madrid) y tiene como título "Los límites de la prefijación". La autora parte de las siguientes dos preguntas para abordar el estudio de los límites de la prefijación respecto de la derivación sufijal y respecto de la composición: qué hace ser a un elemento un prefijo y qué relación existe entre ser un prefijo y situarse a la izquierda de la palabra. Con el fin de dar respuesta a estas cuestiones, la autora explora tres supuestos, enunciados mediante afirmaciones ("los prefijos son afijos"; "los prefijos son raíces"; "los prefijos son preposiciones"). En el primer caso ("los prefijos son afijos"), Josefa Martín García compara la sufijación apreciativa (novelón) y la prefijación gradativa (supernovela). En el segundo caso ("los prefijos son raíces"), la autora contrasta un prefijo prototípico como innegativo con los prefijos adjetivos (macro-, micro-) y los elementos compositivos procedentes de adjetivos relacionales acortados (euro-, eco-, ciber-). Finalmente, en el tercer caso ("los prefijos son 
preposiciones") la autora explora la conexión entre los prefijos y las preposiciones en el ámbito nominal (sinvergüenza, contraventana, entretela, antiarrugas, intercentros). Como conclusión, se propone que los prefijos son afijos que existen como unidades morfológicas pero no forman un grupo homogéneo, pues, aunque comparten una serie de propiedades (posición a la izquierda de la base, elementos no categorizadores, elementos no nucleares en la formación, modificadores semánticos de la base, ausencia de alteración de la forma fonológica de la base), pueden presentar otras que no son compartidas por todos los posibles miembros de la clase (separabilidad de la base, base sintagmática, alcance sobre distintas propiedades de la base, posibilidad de reduplicación, coordinación, polisemia). Por este motivo, la autora considera necesario acudir a la noción de prototipicidad a la hora de definir estas unidades, de manera que los elementos menos prototípicos serán los más proclives al cambio.

Por su parte, en el artículo titulado "La acronimia. Delimitación, rasgos generales y vitalidad de un procedimiento morfológico 'artificial”, José Carlos Martín Camacho (Universidad de Extremadura) compara de forma exhaustiva la acronimia (veroño, viejoven, conspiranoico) con otros procedimientos de formación de palabras próximos: por un lado, con aquellos en los que también se produce una reducción de las bases (acortamiento, siglación y falsa segmentación); por otro, con la composición, ya que a menudo se ha considerado la acronimia como un tipo especial de composición. Para ello, el autor parte de la concepción de la acronimia como un proceso en el que se da "combinación de los significados de las bases en una nueva unidad léxica sin seguir un patrón semántico fijo y fusión lineal de segmentos truncados de los significantes de esas bases sin ajustarse a condicionantes fónicos ni morfémicos" (p. 108). Dado que los límites entre las formaciones comparadas son en ocasiones extremadamente difusos, el autor precisa la definición de acrónimo y la restringe con el fin de excluir todas las formaciones fronterizas, para lo cual enuncia ocho características fundamentales, tanto de tipo formal (por ejemplo, el proceso de reducción debe ser simultáneo al de combinación, algo que no se cumple en bicicarril, pues bici es una forma acortada ya asentada en la lengua), como de tipo semántico. En relación con este último aspecto, por ejemplo, el acrónimo no puede ser una variante diafásica de la base, sino que debe presentar un significado propio y distinto de esta. De este modo, una formación como narraluces (narradores andaluces), considerada acrónimo en otros trabajos, no se ajustaría a la caracterización de acrónimo de Martín Camacho, pues tiene como base un sintagma nominal con el que comparte designación, de manera que la diferencia sería puramente estilística, de carácter diafásico. Se trataría, por tanto, de un caso de acortamiento. Finalmente, en cuanto a la comparación entre composición y acronimia, el autor señala el carácter artificial de este segundo proceso, así como el hecho de que con frecuencia presenta patrones formales y semánticos anómalos y es propia de ámbitos muy concretos (lenguaje literario, discurso periodístico, ámbito comercial y publicitario, ámbito científico-técnico).

Bajo el título "El sufijo -ero locativo-colectivo del español atlántico", el trabajo de Franz Rainer (WU Viena) estudia la formación de sustantivos sufijados mediante -ero con valor locativo (basurero) y, especialmente, colectivo (animalero 'conjunto de animales') tanto en el español de España como en el español de América. El autor comienza describiendo el uso de -ero con valor colectivo en español de América, valor que se atestigua fundamentalmente en la zona del Caribe y Ecuador, para continuar mostrando a partir de fuentes lexicográficas cómo este uso, considerado americano, puede encontrarse también en Andalucía y Canarias. Así, este patrón de sufijación de ero con significado colectivo, que habría surgido de una reinterpretación metonímica del lugar donde abundan ciertas cosas hacia el conjunto de esas cosas, puede entenderse como un rasgo del 
español atlántico, pues el español caribeño habría generalizado una tendencia ya atestiguada en las variedades andaluza y canaria. El trabajo estudia igualmente los nichos semánticos donde se localiza este tipo de formación locativo-colectiva: montón de tierra, con prototipo terrero; montón de basura, con prototipo estercolero y basurero, y abundancia de insectos o bichos, con prototipo hormiguero. Sería este último el que se habría extendido en español caribeño (y Ecuador) a otro tipo de animales (chuchero, iguanero) e incluso a personas (muchachero, mujerero).

En cuanto a las notas, encontramos en primer lugar el trabajo de Peter Stehlík (Universidad Masaryk de Brno), titulado "La sufijación apreciativa: ¿de verdad a medio camino entre la morfología derivativa y la flexiva?", en el que el autor revisa las características de la sufijación apreciativa, con especial atención a aquellas que se han aducido en la bibliografía para considerarla un proceso limítrofe entre derivación y flexión. En su opinión, las similitudes de la derivación apreciativa con la flexión son solo aparentes y superficiales, pues los mismos rasgos caracterizan a la prefijación, por lo que no estaría justificado considerar la derivación apreciativa como un proceso distinto de la derivación en general.

También sobre sufijación apreciativa versa el trabajo de Irene Gil Laforga (Centro de Estudios de la RAE/UCM), en el que, bajo el título "Los límites de la sufijación apreciativa: las formaciones con el sufijo -ón", se estudian las relaciones entre la sufijación derivativa y la sufijación apreciativa a partir del análisis concreto de dos tipos de formaciones en -ón: por una parte, adjetivos posesivos con bases nominales (narizón, barrigona); por otra, sustantivos y adjetivos aumentativos (peliculón, dulzón, simplón). Con el objetivo de dar cuenta de las similitudes formales y semánticas (significado valorativo) existentes entre los dos tipos de formaciones, la autora desarrolla un análisis de carácter léxico-sintáctico en el que se propone un único sufijo -ón, que da lugar a las dos clases de formaciones mencionadas a partir de distintas posiciones en la estructura de la palabra.

El trabajo de Bárbara Marqueta (Universidad de Zaragoza), titulado "Hacia una reconsideración de los tipos de compuestos en español y las propiedades fonológicas de sus constituyentes", parte de la constatación de que las nociones de raíz, stem y palabra no son suficientemente abarcadoras individualmente para dar cuenta de las distintas posibilidades formales de los miembros de un compuesto en español. Así, mientras que el primer constituyente de nadador estrella es una palabra derivada, en el caso de lavaplatos se trata de la combinación de una raíz y una vocal temática y en patilargo de una raíz. La autora presenta una propuesta de caracterización de los constituyentes de las palabras compuestas basada en la idea de que el tema (unidad conceptual) puede tener como correlatos fonológicos bien una base fonológica, bien una palabra fonológica, con propiedades formales diferentes que atañen, por ejemplo, a la existencia o no de diptongo o a la existencia de uno o dos acentos en el compuesto.

Por su parte, el estudio de María Asunción Laguna Álvarez (Universidad de Burgos), titulado "Adjetivos parasintéticos documentados en inventarios de bienes del siglo XVIII", se centra en los adjetivos denominales parasintéticos documentados en inventarios de bienes incluidos en protocolos notariales de la segunda mitad XVIII del Archivo Histórico Provincial de Burgos. La autora presta atención, por un lado, a las formaciones que cuentan con escasa información en las fuentes lexicográficas (abandejada, abaulada, acartelados, ajicarada, atumbado, entre otras); por otro, a los tipos de esquemas y de valores semánticos más frecuentes, así como a los casos de variación (acincelado - cincelado, afeligranado - feligranado, aochavado - ochavado); finalmente, a la existencia de correlación o no entre estas formaciones y participios de verbos recogidos en el DRAE. 
La monografía se cierra con el estudio de Marcial Morera (Universidad de La Laguna), que tiene por título "Clasificación semántica (léxica y gramatical) de las fórmulas de tratamiento (cortesía, apodos, hipocorísticos) del español”. En este trabajo se trazan las relaciones entre elementos aparentemente muy dispares entre sí, como las fórmulas de tratamiento de cortesía (usted, don, señora), los apodos (Mochuelo) y los hipocorísticos (Isa, Blasillo), a partir del concepto de tratamiento subjetivo, que puede implicar alejamiento (como en las fórmulas de tratamiento de cortesía) o bien aproximación, como en apodos e hipocorísticos. Estos dos últimos tipos de expresiones se diferenciarían por el procedimiento que los crea: sustitución en el caso de los apodos y modificación morfológica (con sufijos apreciativos) o fónica (principalmente por acortamiento) en el caso de los hipocorísticos.

Como se puede observar en la síntesis de las distintas contribuciones que acabamos de presentar, el volumen reseñado aborda detalladamente y desde perspectivas a veces complementarias los principales problemas de demarcación que se plantean entre los procesos morfológicos de una lengua como el español. Además, los autores de los distintos trabajos presentan a menudo propuestas de análisis originales y bien fundamentadas en relación con distintos tipos de fenómenos, por lo que el valor de la monografía es doble: como síntesis bien organizada de los problemas identificados habitualmente en la bibliografía a la hora de delimitar los procesos morfológicos y como presentación de propuestas de análisis novedosas en algunos casos. Quizá podría completarse el panorama con algún trabajo en el que se exploren por extenso las fronteras entre la flexión y la derivación. En este sentido, podría profundizarse en algunas zonas de interferencia de la sufijación no apreciativa como son, por ejemplo, el llamado "género derivativo" y los casos de sufijación denominados "transposiciones" (Beard 1995; Spencer 1999): las nominalizaciones, la formación de adjetivos relacionales y la derivación de categorías mixtas como el participio. No obstante, consideramos que la coherencia temática de la mayoría de las contribuciones, así como la cobertura empírica de los fenómenos abordados hacen de Procesos morfológicos. Zonas de interferencia una referencia ineludible a partir de ahora en los estudios sobre morfología del español.

\section{Bibliografía}

Beard, R. (1995): Lexeme Morpheme Base Morphology. Stony Brook, NY: SUNY Press.

Spencer, A. (1999): “Transpositions and argument structure”, in G. Booij y J. van Marle J. (eds.): Yearbook of Morphology 1998. Springer, Dordrecht, pp. 73-101. https://doi.org/10.1007/978-94-017-3720-3 4 